\title{
Studies of Stellar Collapse and Black Hole Formation with the Open-Source Code GR1D
}

\author{
C. D. Ott* and E. O'Connor ${ }^{\dagger}$ \\ *cott@tapir.caltech.edu, TAPIR, California Institute of Technology, Pasadena, CA, 91125, USA \\ †evanoc@tapir.caltech.edu, TAPIR, California Institute of Technology, Pasadena, CA, 91125, USA
}

\begin{abstract}
We discuss results from simulations of black hole formation in failing core-collapse supernovae performed with the code GR1D, a new open-source Eulerian spherically-symmetric general-relativistic hydrodynamics code. GR1D includes rotation in an approximate way (1.5D), comes with multiple finite-temperature nuclear equations of state (EOS), and treats neutrinos in the post-core-bounce phase via a 3-flavor leakage scheme and a heating prescription. We chose the favored $K_{0}=220 \mathrm{MeV}$-variant of the Lattimer \& Swesty (1990) EOS and present collapse calculations using the progenitor models of Limongi \& Chieffi (2006). We show that there is no direct (or "prompt") black hole formation in the collapse of ordinary massive stars $\left(8 M_{\odot} \lesssim\right.$ $M_{\text {ZAMS }} \lesssim 100 M_{\odot}$ ) and present first results from black hole formation simulations that include rotation.
\end{abstract}

Keywords: stellar collapse, neutron stars, black holes, supernovae

PACS: 04.25.D-, 04.40.Dg, 97.10.Kc, 97.60.Bw, 97.60.Jd, 97.60.Lf, 26.60.Kp

\section{INTRODUCTION}

Core-collapse supernova explosions are the dramatic events heralding massive star death in core collapse. All stars in the zero-age-main-sequence (ZAMS) mass range from $\gtrsim 8 M_{\odot}$ to $\sim 100 M_{\odot}$ undergo core collapse at the end of their life, but not all core collapse events result in a core-collapse supernova explosion. In any star of the mass range under consideration, core collapse separates the stellar core into subsonically collapsing inner core and supersonic outer core. The collapse of the former is stabilized by the stiffening of the nuclear equation of state (EOS) near nuclear density. Core bounce occurs, launching a hydrodynamic shock into the still infalling outer core. This prompt shock fails to blow up the star and is forced into stall by the dissociation of accreting iron-group nuclei and neutrino losses from the postshock region. The shock must be revived for core collapse to result in an explosion. The precise mode of revival provided by the much sought-after supernova mechanism is still uncertain (e.g., $[1,2,3,4,5,6])$, and in a finite (though unknown) fraction of massive stars, it must fail completely to drive an explosion, or, at least fail to unbind the entire star so that significant fallback accretion occurs [7]. In the former case, a black hole (BH) inevitably forms within a few seconds as the stellar mantle accretes onto the protoneutron star (PNS; Collapsar type I [8]), while in the latter, a neutron star initially survives but may be pushed over its mass limit by fallback accretion (Collapsar type II $[8,9]$ ). Both are considered as potential scenarios leading to a long gamma-ray burst (GRB) $[8,10]$.

BH formation in the core collapse context has been studied with full generalrelativistic (GR) Boltzmann neutrino radiation-hydrodynamics codes in spherical 
symmetry without rotation (e.g., $[11,12,13]$ and references therein), but due to the computational cost of these high-fidelity simulations, only few models have been simulated. Multi-dimensional simulations of $\mathrm{BH}$ formation remain to be performed with appropriate microphysics and progenitor models, but see [14] for an exploratory work with simplified physics.

We have recently implemented the new code GR1D, an Eulerian sphericallysymmetric code for stellar collapse and BH formation [15]. GR1D is open-source and may be downloaded from http: / / www. stellarcollapse.org. GR1D, in its present version, does not implement the full radiation transport formalism, but instead relies on an approximate, yet extremely computationally efficient leakage scheme for neutrino transport (e.g., $[16,17])$ and a simple prescription for neutrino heating. Moreover, GR1D implements an approximate way of including rotation in spherical symmetry a variant of which is used in stellar evolutionary calculations (e.g., [18]). GR1D's computational efficiency allows us to perform hundreds of model simulations in little time to explore systematically the conditions for $\mathrm{BH}$ formation in massive stars and the parameter space in ZAMS mass, metallicity, and rotation where $\mathrm{BH}$ formation may be the dominant outcome of stellar collapse. Detailed results of such an extensive study will be reported in [19].

In the following, we discuss some details of the GR1D code, then present simulation results highlighting the fact that any $\mathrm{BH}$ forming core collapse passes through a PNS phase and is never "direct". We then show first results from simulations of rotating BH formation.

\section{THE GR1D CODE}

GR1D follows the $3+1$ approach to numerical relativity, slicing 4D spacetime into 3D spacelike hypersurfaces along a timelike normal (e.g., [20]). This introduces two gauge quantities, the lapse function $\alpha$ and the shift vector $\vec{\beta}$ whose choices are not a priori fixed. The lapse controls how time changes between two consecutive slices while the shift describes how coordinates change from one slice to the next. In GR1D, we adopt spherical symmetry and the polar-slicing, radial-gauge choice (e.g., [21]) on an Eulerian

grid, resulting in $\vec{\beta}=0$ and a Schwarzschild-like invariant line element of the form (assuming $c=G=M_{\odot}=1$ here and in the following),

$$
d s^{2}=-\alpha(r, t)^{2} d t^{2}+X(r, t)^{2} d r^{2}+r^{2} d \Omega^{2},
$$

where the lapse $\alpha$ and the metric function $X$ are functions of a metric potential $\Phi(r, t)$ and of the enclosed gravitational mass $M_{\text {grav }}(r, t)=m(r, t)$,

$$
\alpha(r, t)=\exp [\Phi(r, t)], \quad X(r, t)=\left(1-\frac{2 m(r, t)}{r}\right)^{-1 / 2} .
$$

The detailed form of $\Phi(r, t)$ can be found in [15, 21]. Here we point out only that the choice of $\Phi$ ensures that $\alpha$ is singularity-avoiding and, hence, drops to very small values when a physical singularity forms, thus minimizing its evolution with coordinate time. 
It is important to note that the metric function $X$ becomes singular at $r=2 M$ and thus forbids numerical evolution beyond $\mathrm{BH}$ formation in this gauge (a feature in common with many other $1 \mathrm{D}$ codes $[11,13])$.

GR1D's hydrodynamics module follows the flux-conservative Valencia formulation of GR hydrodynamics in the form of [22]. The scheme is semi-discrete in space and is finite-volume with piecewise-parabolic reconstruction of interface values and employs the HLLE Riemann solver [23]. Time discretization is handled via 2nd- or 3rd-order Runge-Kutta integrators using the Method of Lines [24].

A specialty of GR1D is its approximate inclusion of rotation in spherical symmetry (1.5D). This is accomplished by solving an advection equation for the angular momentum and including an angularly averaged centrifugal term in the radial momentum equation, in the Lorentz factor, and in curvature terms to account for centrifugal force, angular momentum flux, and rotational energy [15].

GR1D operates with a general EOS interface and has been tested with variants of the Lattimer-Swesty EOS [25] and the H. Shen EOS [26] tables of which we make available for download at http: / / stellarcollapse.org and describe in [15].

Deleptonization and neutrino transport are handled by GR1D differently in the prebounce and postbounce phases. In the former, we employ the simple parameterization of the electron fraction $Y_{e}$ as a function of rest-mass density $\rho$ put forth by [27]. In the latter, we use a 3-flavor $\left(v_{e}, \bar{v}_{e}\right.$, and $\left.v_{x}=\left\{v_{\mu}, \bar{v}_{\mu}, v_{\tau}, \bar{v}_{\tau}\right\}\right)$ energy-averaged leakage scheme constructed along the lines of [16, 17] and described in [15]. It yields energy-integrated neutrino luminosities that are within $\sim 20 \%$ of those predicted by full Boltzmann transport calculations. Neutrino heating by charged-current absorption of $v_{e}$ and $\bar{v}_{e}$ is handled via

$$
Q_{v_{i}}^{\text {heat }}(r)=f_{\text {heat }} \frac{L_{v_{i}}(r)}{4 \pi r^{2}} \sigma_{\text {heat }, v_{i}} \frac{\rho}{m_{u}} X_{i}\left\langle\frac{1}{F_{v_{i}}}\right\rangle e^{-2 \tau_{v_{i}}},
$$

where $L_{v_{i}}(r)$ is the luminosity as set by the energy leakage rate interior to radius $r$, $\sigma_{\text {heat }, v_{\mathrm{i}}}$ is the energy-averaged absorption cross section, $m_{u}$ is the atomic mass unit, $X_{i}$ is the mass fraction of the absorbing particle (proton or neutron), and $\left\langle 1 / F_{v_{i}}\right\rangle$ is the mean inverse flux factor which we approximate analytically as a function of the optical depth $\tau$ by comparing to angle-dependent radiation transport calculations [3, 15]. The factor $e^{-2 \tau_{v_{i}}}$ is used to attenuate heating at high optical depths where neutrinos and matter are in equilibrium. $f_{\text {heat }}$ is a scaling factor that may be used to dial in higher heating rates, but is set to 1 here, causing explosions to fail in all models. Once the heating rate is computed, the luminosity is reduced accordingly to maintain energy conservation.

\section{NO DIRECT BLACK HOLE FORMATION IN CORE COLLAPSE}

The idea prevails that stars in the upper half of the $\sim 8 M_{\odot}$ to $\sim 100 M_{\odot}$ mass range collapse to BHs directly. This is incorrect, but, by an unfortunate choice of words in recent work $[8,28]$ that can easily be misunderstood, has become widely believed.

According to Thorne's Hoop Conjecture [29], a BH forms when a given amount of mass-energy collapses through its own Schwarzschild radius $R_{\mathrm{S}}=2 M$ (in geometric units), hence must achieve a compactness $M / R>0.5$. If prompt $\mathrm{BH}$ formation were to occur in core collapse, the collapsing core (or parts of it) must reach this level of 


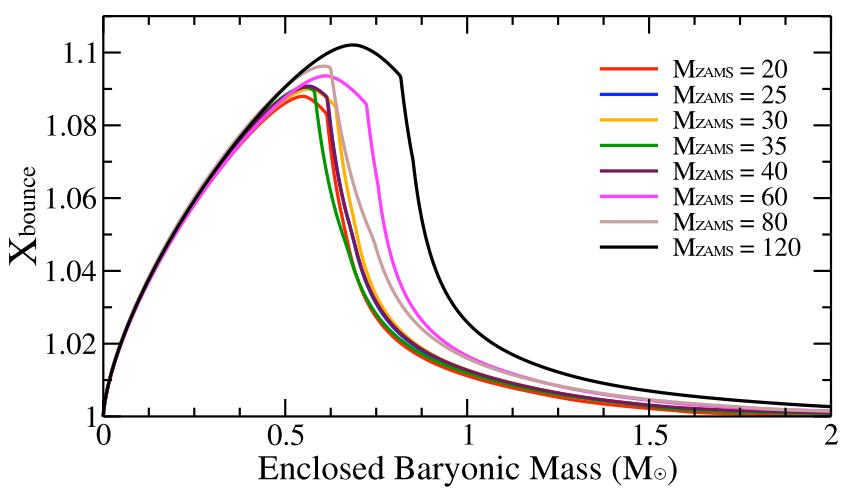

FIGURE 1. Metric coefficient $X=1 / \sqrt{1-2 m / r}$ as a function of enclosed baryonic mass at the time of core bounce and greatest inner-core compactness. We plot $X$ for GR1D collapse simulations of models from [30] with ZAMS masses from 20 to $120 M_{\odot}$. If a $\mathrm{BH}$ were to form, $X$ would diverge. Due to the universality of core collapse, inner core masses vary only little with progenitor and the compactness at bounce stays moderate independent of progenitor mass / model. There is no prompt formation of BHs. While GR1D's treatment of deleptonization during collapse is approximate, it agrees well qualitatively and quantitatively with more quantitatively accurate simulations (e.g., [27]).

compactness prior to or at bounce. In the following, we demonstrate that this is never the case for stars in the above mass range and explain why.

Goldreich \& Weber [31] and Yahil [32] demonstrated analytically what has been confirmed numerically numerous times: In collapse, the iron core separates into the homologously ( $v \propto r$, in sonic contact) infalling inner core (IC) and the supersonically collapsing outer core. It is only the inner core that plunges to nuclear density and significant compactness in the final phase of core collapse and it is essentially its mass $\left(M_{\mathrm{IC}}\right)$ that must be pushed below its Schwarzschild radius to make a $\mathrm{BH}$.

$M_{\mathrm{IC}}$ at bounce is proportional to $Y_{e, \mathrm{IC}}^{2}\left(1+\eta s_{\mathrm{IC}}^{2}\right)$ [33], where $\eta \approx 0.1$ and where $Y_{e, \mathrm{IC}}$ and $s_{I C}$ are the mean $Y_{e}$ and specific entropy of the inner core at bounce, respectively. Both quantities are coupled through electron capture, neutrino transport, and the equalibration of neutrinos and matter above trapping density. The physics governing $Y_{e, I C}$ and $s_{\text {IC }}$ during collapse is general and independent of the conditions prevailing in a particular collapsing star $[27,1]$. Hence, core collapse is universal, rather independent of initial conditions, and $M_{\mathrm{IC}}$ falls into the range of $\sim 0.4-0.6 M_{\odot}$ in the nonrotating case $^{1}[35,34]$. The Hoop Conjecture would require this mass to be compressed into $1.3-1.8 \mathrm{~km}$, which even for the softest plausible nuclear EOS (e.g., the $K_{0}=180 \mathrm{MeV}$ variant of the Lattimer-Swesty EOS $[25,36]$ ) does not occur before or at bounce.

In Fig. 1, we plot the metric coefficient $X$ (Eq. 2) at the time of core bounce and as a function of enclosed baryonic mass. $X$ is shown for a set of solar-metallicity models in the $20-120 M_{\odot}$ mass range. These are drawn from the Limongi \& Chieffi [30]

\footnotetext{
${ }^{1}$ Rotation can increase the size of the homologous region and, hence, $M_{\mathrm{IC}}$ significantly, but also limits inner core compactness [34].
} 


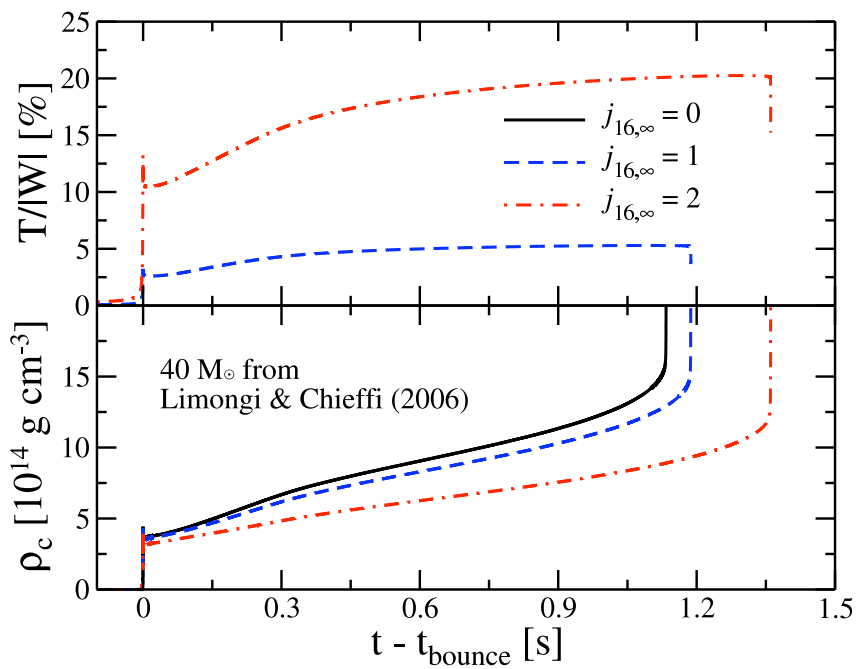

FIGURE 2. Example results from GR1D simulations of a 40- $M_{\odot}$ progenitor of [30] set up to spin with asymptotic specific angular momenta $j_{16}=\{0,1,2\}$ (see text for details). Bottom: Evolution of the central density as a function of postbounce time. Rotational support leads to a lower accretion rate, slower PNS contraction, and a larger maximum PNS mass. At BH formation, the PNS baryonic mass is $2.37,2.39$, and $2.46 M_{\odot}$, for the $j_{16}=0,1$, and 2 cases, respectively. Top: Ratio of rotational kinetic energy $T$ to gravitational binding energy $|W|$ in the two spinning simulations. In both models, a local maximum is reached at bounce and is followed by a secular increase in the postbounce phase as the PNS core contracts and spins up. The $j_{16}=2$ run reaches values of $T /|W|$ that may make it susceptible to a secular nonaxisymmetric instability [38] before BH formation. Both models may develop a low- $T /|W|$ corotation-type nonaxisymmetric instability (e.g., [39]).

model set (using the mass-loss rates of [37]) and collapsed with GR1D without rotation, using the Lattimer-Swesty $K_{0}=220 \mathrm{MeV}$ (LS220) EOS, and an analytic fit to the deleptonization trajectory $Y_{e}(\rho)$ obtained by [27]. The figure clearly underpins the universality of core collapse and demonstrates that none of the collapse models come even close to $B H$ formation before or at bounce (for which $X \gg 1$ ). Variations of the $X(m)$ profiles with progenitor mass are due primarily to larger inner core masses owing to higher temperatures/entropies in more massive progenitors. In a full radiationhydrodynamics treatment, one would expect these variations to be even smaller due to increased deleptonization in hotter cores.

\section{THE FORMATION OF SPINNING BLACK HOLES}

We perform 1.5D rotating collapse simulations of the $40-M_{\odot}$ progenitor of [30] with the LS220 EOS. Rotation is added when the peak collapse velocity of the core reaches $1000 \mathrm{~km} \mathrm{~s}^{-1}$ via the rotation law $\Omega(r)=\Omega_{0}\left(1+r^{2} / A^{2}\right)^{-1}$. A is set to the radius at which the enclosed baryonic mass is $1 M_{\odot}$, resulting in nearly uniform rotation throughout 
the inner core [40]. We perform three calculations with $\Omega_{0}=\{0,0.9,1.8\} \mathrm{rad} \mathrm{s}^{-1}$, corresponding to specific angular momenta at infinity in units of $10^{16} \mathrm{~cm}^{2} \mathrm{~s}^{-1}$ of $j_{16, \infty}=$ $\{0,1,2\}$.

In the lower panel of Fig. 2, we show the time evolution of the central density in the three calculations as a function of postbounce time. The nonrotating model forms a $\mathrm{BH}$ at $\sim 1.14 \mathrm{~s}$ and has a maximum baryonic (gravitational) PNS mass of $\sim 2.37 M_{\odot}$ $\left(\sim 2.19 M_{\odot}\right)$. In the rotating models, centrifugal support reduces the accretion rate and slows down the contraction of the PNS. This alters the PNS structure and leads to an onset of PNS collapse at later times (scaling roughly with $\Omega_{0}^{2}$ ) and at lower central densities. The times of $\mathrm{BH}$ formation are $\sim 1.19 \mathrm{~s}$ and $\sim 1.36 \mathrm{~s}$ in the $j_{16, \infty}=1$ and $j_{16, \infty}=2$ model, respectively. Interestingly, rotational support has only a small effect on the maximum PNS masses in the models presented here. This is due primarily to the fact that the PNS cores in our models are uniformly spinning. A significant increase of the maximum PNS mass is expected only in differentially rotating PNSs [41]. We find baryonic (gravitational) PNS masses at BH formation of $\sim 2.39 M_{\odot}\left(\sim 2.22 M_{\odot}\right)$ and $\sim 2.46 M_{\odot}\left(\sim 2.30 M_{\odot}\right)$ in the two spinning models.

The top panel of Fig. 2 depicts the time evolution of the ratio of rotational kinetic energy $T$ to gravitational energy $|W|$ in the spinning models. In both, $T /|W|$ increases secularly after bounce as the PNS accretes and contracts and reaches a maximum value before the onset of PNS collapse of $\sim 0.20(\sim 0.05)$ in the $j_{16, \infty}=2\left(j_{16, \infty}=1\right)$ model. Both models stay below the threshold $T /|W|_{\text {dyn }} \approx 0.27$ for the high- $T /|W|$ dynamical nonaxisymmetric instability (e.g., [42]). The rapidly spinning PNS of the $j_{16, \infty}=2$ model stays above $T /|W|_{\text {sec }} \approx 0.14$ for $\sim 1 \mathrm{~s}$, a time likely sufficiently long for a secular (gravitational-wave or viscosity driven) nonaxisymmetric instability to arise in 3D (e.g., [38]), redistributing/radiating angular momentum and thus effectively limiting the PNS core spin. In addition, both models may be susceptible to shear instabilities that may generate nonaxisymmetric structure (e.g., [39]) and/or magnetic flux (e.g., [43]) and redistribute angular momentum. In our present $1.5 \mathrm{D}$ calculations such processes and instabilities are absent and the $\mathrm{BH}$ formed in the rapidly spinning $j_{16, \infty}=2$ model has an initial spin parameter $a^{\star}=J / M_{\text {grav }}^{2}$ of $\sim 0.81$. Its more slowly spinning $j_{16, \infty}=1$ counterpart forms a BH with $a^{\star} \sim 0.41$.

\section{DISCUSSION}

In this contribution to the proceedings of the 10th International Symposium on the Origin of Matter and Evolution of the Galaxies (OMEG10), we have highlighted results of a small set of stellar collapse and $\mathrm{BH}$ formation simulations carried out with the new open-source 1.5D code GR1D. We numerically demonstrated what has long been known on the basis of analytic arguments [31, 32], namely that a $\mathrm{BH}$ is never formed promptly in the core collapse of ordinary massive stars with masses between $\sim 8 M_{\odot}$ and $\sim 100 M_{\odot}$. Any core collapse event, if ultimately resulting in $\mathrm{BH}$ formation or not, passes through a protoneutron star phase in which neutrinos and, quite likely, gravitational waves, are emitted for at least a few hundred milliseconds. Direct subsidence into a BH is possible only in much more massive stars that become radially unstable before forming a hydrostatic iron core (e.g., [44]), provided they do not experience a pair-instability 
driven thermonuclear disruption instead of collapse.

Massive stars, in particular those favored as the progenitors of long GRBs, may have rapidly spinning cores [10] that will make spinning BHs. Using GR1D's 1.5D rotation feature, we have performed a first small set of spinning $\mathrm{BH}$ formation simulations. Rotational effects increase the time to $\mathrm{BH}$ formation significantly (and, thus, allow a potential explosion mechanism more time to operate!), but, due to almost uniform rotation in the PNS core, have a much smaller impact on the maximum PNS mass. We also find that the rotation rate $T /|W|$ of PNSs increases significantly during the postbounce accretion and contraction phase. PNSs with early postbounce values of $T /|W|$ below the thresholds for secular or dynamical rotational instabilities may surpass these within a few hundred milliseconds. Nonaxisymmetric deformation and the associated angular momentum redistribution and/or emission of angular momentum in gravitational waves may put a natural limit on the maximum PNS spin and, in consequence, on the birth spin of BHs.

Stellar-mass BH formation is a process that occurs frequently in the universe and core collapse is its natural site. As observations suggest ([45] and references therein), it may be the generic ultimate outcome of core collapse in stars more massive than $\sim 20 M_{\odot}$. In the context of the core-collapse supernova-GRB connection, $\mathrm{BH}$ formation is a necessary ingredient for the collapsar scenario to work (e.g., $[8,10])$. The current theoretical understanding of core-collapse supernovae and $\mathrm{BH}$ formation is still only partial. Ultimately, it will be necessary to establish a firm quantitative mapping between ZAMS conditions (mass, metallicity, angular momentum) and the outcome of stellar collapse. This is a problem as much in stellar evolution as in core-collapse supernova theory and will require advances in both fields.

With the GR1D code we have created an open-source tool that allows us and others to study $\mathrm{BH}$ formation in failing core-collapse supernovae and to investigate its systematics and the characteristics of nascent BHs with variations in presupernova stellar structure and rotational configuration. While a quantitatively robust mapping to ZAMS conditions may not be possible on the basis of currently available stellar evolutionary models, robust qualitative features and trends can be derived in parameter studies [19].

\section{ACKNOWLEDGMENTS}

It is a pleasure to thank the organizers of the OMEG10 symposium. Furthermore, we acknowledge helpful and stimulating conversations with W. D. Arnett, A. Burrows, M. Duez, T. Fischer, C. Fryer, J. Lattimer, C. Meakin, F. Peng, C. Reisswig, E. Schnetter, H. Shen, U. Sperhake, K. Sumiyoshi, F. Timmes, K. Thorne, and H. Toki. CDO is supported in part by the National Science Foundation under grant numbers AST-0855535 and OCI-0905046. EOC is supported in part through a post-graduate fellowship from the Natural Sciences and Engineering Research Council of Canada (NSERC). Computations were performed on the Louisiana Optical Network Infrastructure computer systems under allocation loni_numrel04. 


\section{REFERENCES}

1. H.-T. Janka, K. Langanke, A. Marek, G. Martínez-Pinedo, and B. Müller, Phys. Rep. 442, 38 (2007).

2. A. Marek, and H.-T. Janka, Astrophys. J. 694, 664 (2009).

3. C. D. Ott, A. Burrows, L. Dessart, and E. Livne, Astrophys. J. 685, 1069 (2008).

4. A. Burrows, E. Livne, L. Dessart, C. D. Ott, and J. Murphy, Astrophys. J. 640, 878 (2006).

5. A. Burrows, L. Dessart, E. Livne, C. D. Ott, and J. Murphy, Astrophys. J. 664, 416 (2007).

6. I. Sagert, T. Fischer, M. Hempel, G. Pagliara, J. Schaffner-Bielich, A. Mezzacappa, F. Thielemann, and M. Liebendörfer, Phys. Rev. Lett. 102, 081101 (2009).

7. F. X. Timmes, S. E. Woosley, and T. A. Weaver, Astrophys. J. 457, 834 (1996).

8. A. Heger, C. L. Fryer, S. E. Woosley, N. Langer, and D. H. Hartmann, Astrophys. J. 591, 288 (2003).

9. W. Zhang, S. E. Woosley, and A. Heger, Astrophys. J. 679, 639 (2008).

10. S. E. Woosley, and J. S. Bloom, Ann. Rev. Astron. Astrophys. 44, 507 (2006).

11. T. Fischer, S. C. Whitehouse, A. Mezzacappa, F.-K. Thielemann, and M. Liebendörfer, Astron. Astrophys. 499, 1-15 (2009).

12. K. Sumiyoshi, S. Yamada, H. Suzuki, and S. Chiba, Phys. Rev. Lett. 97, 091101 (2006).

13. K. Sumiyoshi, S. Yamada, and H. Suzuki, Astrophys. J. 667, 382 (2007).

14. Y.-I. Sekiguchi, and M. Shibata, Phys. Rev. D. 71, 084013 (2005).

15. E. O'Connor, and C. D. Ott, Class. Quant. Grav. 27, 114103 (2010).

16. M. Ruffert, H.-T. Janka, and G. Schäfer, Astron. Astrophys. 311, 532 (1996).

17. S. Rosswog, and M. Liebendörfer, Mon. Not. Roy. Astron. Soc. 342, 673 (2003).

18. A. Heger, N. Langer, and S. E. Woosley, Astrophys. J. 528, 368 (2000).

19. E. O'Connor, and C. D. Ott, in preparation (2010).

20. M. Alcubierre, Introduction to 3+1 Numerical Relativity, Oxford University Press, 2008.

21. E. Gourgoulhon, Astron. Astrophys. 252, 651 (1991).

22. J. V. Romero, J. M. Ibanez, J. M. Marti, and J. A. Miralles, Astrophys. J. 462, 839 (1996).

23. B. Einfeldt, "On Godunov type methods for the Euler equations with a general equation of state," in Shock tubes and waves; Proceedings of the Sixteenth International Symposium, Aachen, Germany, July 26-31, 1987 (A89-12876 03-34). Weinheim, Germany, VCH Verlagsgesellschaft mbH, 1988, 1988, pp. 671-676.

24. J. M. Hyman, The method of lines solution of partial differential equations, Tech. Rep. COO-3077139, ERDA Mathematics and Computing Laboratory, Courant Institute of Mathematical Sciences, New York University (1976).

25. J. M. Lattimer, and F. D. Swesty, Nucl. Phys. A 535, 331 (1991).

26. H. Shen, H. Toki, K. Oyamatsu, and K. Sumiyoshi, Prog. Th. Phys. 100, 1013 (1998).

27. M. Liebendörfer, Astrophys. J. 633, 1042 (2005).

28. S. E. Woosley, A. Heger, and T. A. Weaver, Rev. Mod. Phys. 74, 1015 (2002).

29. K. S. Thorne, Nonspherical Gravitational Collapse-A Short Review, 1972, pp. 231-258.

30. M. Limongi, and A. Chieffi, Astrophys. J. 647, 483 (2006).

31. P. Goldreich, and S. V. Weber, Astrophys. J. 238, 991 (1980).

32. A. Yahil, Astrophys. J. 265, 1047 (1983).

33. A. Burrows, and J. M. Lattimer, Astrophys. J. 270, 735 (1983).

34. H. Dimmelmeier, C. D. Ott, A. Marek, and H.-T. Janka, Phys. Rev. D. 78, 064056 (2008).

35. W. R. Hix, O. E. Messer, A. Mezzacappa, M. Liebendörfer, J. Sampaio, K. Langanke, D. J. Dean, and G. Martínez-Pinedo, Phys. Rev. Lett. 91, 201102 (2003).

36. A. W. Steiner, J. M. Lattimer, and E. F. Brown, ArXiv:1005.0811 [astro-ph] (2010).

37. T. Nugis, and H. J. G. L. M. Lamers, Astron. Astrophys. 360, 227-244 (2000).

38. D. Lai, and S. L. Shapiro, Astrophys. J. 442, 259 (1995).

39. A. L. Watts, N. Andersson, and D. I. Jones, Astrophys. J. Lett. 618, L37 (2005).

40. C. D. Ott, A. Burrows, T. A. Thompson, E. Livne, and R. Walder, Astrophys. J. Suppl. Ser. 164, 130 (2006).

41. T. W. Baumgarte, S. L. Shapiro, and M. Shibata, Astrophys. J. Lett. 528, L29 (2000).

42. N. Stergioulas, Liv. Rev. Rel. 6, 3 (2003).

43. M. Obergaulinger, P. Cerdá-Durán, E. Müller, and M. A. Aloy, Astron. Astrophys. 498, 241 (2009).

44. K. Nakazato, K. Sumiyoshi, and S. Yamada, Astrophys. J. 645, 519 (2006).

45. S. J. Smartt, Ann. Rev. Astron. Astroph. 47, 63 (2009). 